\title{
Orientation-specific mechanisms \\ in the human visual system
}

SEYMOUR M. ANTELMAN, ${ }^{2}$ RONALD OLSON, AND J. ORBACH MICHAEL REESE HOSPITAL AND MEDICAL CENTER

Thresholds for the identification of test lines were elevated when their orientation matched that of adapting lines. This proved to be true when the adapting field was moving or stationary. These results are discussed in relation to the conception of orientation-specific detectors postulated by Hubel and Wiesel.

The microelectrode work of Hubel and Wiesel $(1962,1965)$ in infrahuman species has suggested that a given cell in striate cortex is highly selective with respect to the stimulus patterns to which it responds. Rectangular patches and spots of light, to which optic nerve and lateral geniculate cells are maximally sensitive, were relatively ineffective in stimulating cells in striate cortex. These cells were found to be narrowly tuned to orientation of line and direction of movement. Maximal cortical responses were obtained with lines moving in a direction perpendicular to the orientation of the line. An analysis of relative cell frequency suggested that the cells specific to vertical, horizontal, and oblique lines were approximately equally common.

The present study represents an initial attempt to extend to man the analysis of orientation-specific mechanisms in the visual system. We argued (as did Sekuler and Ganz, 1963, for movement-specific mechanisms) that it should be possible to selectively satiate or adapt elements responsive to a particular orientation so that the threshold of subsequently presented test lines would be raised. We are concerned with the identification of test-line orientation, not merely with the detection of the line.

\section{Subjects}

Twenty college men and women, divided into two groups of 10 , participated in the two experiments. All Ss had normal or corrected vision and were naive with respect to the experimental hypotheses.

\section{Apparatus and Stimulus Conditions}

A three-field mixing tachistoscope manufactured by Scientific Prototype in New York was used. With this instrument, stimuli were presented under constant illumination for a variable exposure time. In each of the two experiments, three adapting fields were used. Each adapting field consisted of parallel black lines on a white background and each was characterized by a particular orientation of the lines, vertical, 45-deg oblique, or 135-deg oblique. Similarly, the test line, black on a white background, was vertical, 45-deg oblique, or 135-deg oblique. Adapting and test fields were viewed through the binocular viewing mask of the tachistoscope.

For Experiment 1, the adapting stimulus moved horizontally back and forth during viewing. (The use of a moving adapting field represents an attempt to adapt a maximal number of cells responding to a given orientation within the visual field of the $S$.) The stimulus card was mounted in a holder that was driven by a motor horizontally back and forth at the rate of $1 \mathrm{in.} \mathrm{every}$ $11 / 2 \mathrm{sec}_{3}$. The holder was mounted in a lightproof extension at tached behind the adapting field.

For Experiment 2, the adapting lines were stationary. To
Table 1

Mean Thresholds (Msec) and SDs of a Test Line that Followed an Adapting Field of Moving Lines $(N=10)$.

\begin{tabular}{lllr}
\hline Adapting Lines & Test Line & Mean & SD \\
\hline Vertical & Vertical & 7.77 & 1.42 \\
& 135 deg Oblique & 6.40 & .83 \\
& 45 deg Oblique & 6.43 & 1.18 \\
135 deg Oblique & Vertical & 6.07 & .96 \\
& 135 deg Oblique & 8.17 & 1.42 \\
45 deg Oblique & 45 deg Oblique & 5.97 & 1.41 \\
& Vertical & 6.50 & 1.21 \\
& 135 deg Oblique & 6.37 & .95 \\
& 45 deg Oblique & 7.43 & 1.05 \\
\hline
\end{tabular}

equate viewing distances of adapting and test fields, an extension was attached behind the test field. The standard viewing distance of each field of the tachistoscope was 48 in. The extensions lengthened the viewing distance to 51 in. Thus, the visual field subtended angles of $5 \mathrm{deg} 58 \mathrm{~min}$ vertical and $8 \mathrm{deg} 20 \mathrm{~min}$ horizontal at the retina.

\section{Procedure}

\section{EXPERIMENT 1}

Ss were presented with one of the three moving adapting fields for $4 \mathrm{sec}$ followed by one of the three test fields. There were nine possible combinations of adapting and test fields. Each combination was exposed three times and the 27 resulting combinations were arranged in random sequence. At the start of each of the 27 presentations, the exposure time of the test line was set at a subthreshold level. The exposure time was raised in steps of $1 \mathrm{msec}$ until the $S$ identified the orientation of the test line. This constituted the ascending scale of the method of limits. Each of the 27 combinations was discontinued when the $S$ was able to identify correctly the orientation of the test stimulus.

Between presentation of adapting and test fields, a lighted blank field was presented for $200 \mathrm{msec}$. The use of light during this interval between stimuli served to raise the absolute threshold for the perception of the stimuli to an intensity level at which the instrument was reliable. After the experimental procedure was explained to the $S$, he was given a number of practice trials using a horizontal line as test stimulus. This enabled the $E$ to estimate the threshold for the correct identification of the test line.

To insure that responses were made at just suprathreshold levels, Ss were asked to refrain from guessing. Between trials, Ss were instructed to look away from the instrument and to keep their eyes open, so that they would not become dark-adapted. The entire experiment was conducted under binocular viewing conditions.

\section{Results}

The results presented in Table 1 suggest that thresholds for the identification of test lines were highest when they matched the adapting lines in orientation. An analysis of variance yielded significantly different mean thresholds for the nine combinations of adapting and test fields $(F=7.63, \mathrm{df}=8 / 72, \mathrm{p}<.01)$.

Planned comparisons using the Newman-Keuls method (Winer. 
1962) were computed for all possible differences between means. When both adapting and test lines matched, the obtained mean thresholds for perception of the test stimuli were almost always significantly higher $(\mathrm{p}<.05)$ than when adapting and test lines differed in orientation. Exceptions to this generalization were found when either a vertical or 45-deg oblique adapting field was paired with a 135-deg oblique test line. There were no significant differences in mean thresholds between test lines paired with matching adapting fields. Neither were any threshold differences found between test lines paired with dissimilar adapting fields.

\section{Comment}

The resuits of the present study indicate that it is possible to adapt selectively orientation-specific mechanisms in the human visual system.

It might be argued that threshold was raised when test and adapting lines matched because the test line stimulated retinal elements that had been fatigued a moment earlier by an adapting line. Since the adapting field moved, we would tend to discount this possibility, because the exact coincidence of an adapting and test line would occur very infrequently.

Is movement of the adapting lines necessary to elevate the threshold of test lines matching the adapting field? We chose to move the adapting field in order to adapt a maximal number of cells responsive to a given orientation. In the next experiment, we employed stationary adapting fields.

Because the test and adapting fields were at slightly different viewing distances, the retinal image of the test line was not as sharp as it might have been. This could not account for the differential threshold; nevertheless, we equated viewing distance of test and adapting lines in the next experiment.

\section{EXPERIMENT 2}

Procedure

Ss were presented with one of two adapting fields, vertical and 135-deg oblique, for $4 \mathrm{sec}$. This was followed by one of the three test fields, vertical, 135-deg oblique, and 45-deg oblique. For this experiment, the adapting lines were stationary. The test lines were positioned to stimulate a portion of the retina that had not been previously adapted by any of the stationary lines in the adapting field. 3

There were six possible combinations of adapting and test fields. Each combination was repeated three times and the 18 resulting combinations were presented in random sequence. As in Experiment 1, the ascending scale of the method of limits was used to estimate the threshold of the test line. Each combination was discontinued when the $S$ was able to identify correctly the orientation of the test line. Other aspects of procedure and instructions to the $\mathrm{S}$ were similar to those of Experiment 1.

The apparatus used in this experiment was modified so that viewing distances of adapting and test fields were identical. It should be recalled that, for Experiment 1, an extension was mounted on the tachistoscope so that the adapting field was viewed at $51 \mathrm{in.}$ For the present experiment, a similar extension was provided for the test field so that both fields would be viewed at $51 \mathrm{in}$.

\section{Results}

Table 2 presents the mean thresholds and standard deviations for the identification of each of the three test lines in combination with the two adapting fields. An analysis of variance indicates that these mean thresholds are significantly different from one another $(F=19.36$, df $=5 / 45, p<.01)$. The NewmanKeuls planned-comparison technique (Winer, 1962) was used to pinpoint the exact location of these differences. As in Experiment 1, when adapting and test stimuli matched in
Table 2

Mean Thresholds (Msec) and SDs of a Test Line that Followed an Adapting Field of Stationary Lines $(N=10)$.

\begin{tabular}{llll}
\hline Adapting Lines & Test Lines & Mean & SD \\
\hline Vertical & Vertical & 13.80 & 2.03 \\
& 135 deg Oblique & 12.70 & 2.30 \\
135 deg Oblique & 45 deg Oblique & 11.17 & 2.79 \\
& Vertical & 11.27 & 2.59 \\
& 135 deg Oblique & 15.63 & 2.35 \\
& 45 deg Oblique & 10.67 & 2.93 \\
\hline
\end{tabular}

orientation, their mean thresholds were significantly higher $(p<.01)$ than when they differed in orientation. There were no significant differences in mean threshold between test lines paired with matching adapting fields, or between test lines paired with dissimilar adapting fields.

\section{Comment}

The results of this experiment suggest that movement of the adapting field is not necessary to obtain the differential thresholds seen in Experiment 1. The threshold of a test line was elevated even when the matching adapting lines were stationary.

It will be noted that the mean thresholds for the correct identification of the test lines in Experiment 2 are considerably higher than those obtained in Experiment 1. This increase in threshold was probably due to the fact that the test lines at a viewing distance of 51 in. were exposed $3 \mathrm{in}$. further from the source of illumination.

\section{GENERAL DISCUSSION}

Assuming that there are narrowly tuned orientation detectors in the human visual system, the data of both experiments indicate that it is possible to adapt these detectors differentially to elevate the threshold for the identification of orientation.

The data presented in Experiment 2, suggesting that movement of the adapting lines was not necessary to elevate the threshold of the matching test line, need to be considered further. Hubel and Wiesel (1965) found that certain cortical cells in the cat, which they termed "simple," respond to straight-line stimuli when these are positioned at exactly the region defined by excitatory and inhibitory areas. A second group of cortical cells, termed "complex," respond to a properly oriented line regardless of precise position, provided that the stimulus is within the cell's receptive field of 5 or $10 \mathrm{deg}$. Unlike "simple" cells, "complex" cells show a steady response to moving lines.

In order to insure the adaptation of the "simple" and "complex" cells responding to a particular orientation, adapting lines were moved across the visual field. Had the adapting field been stationary, there was the possibility that "simple" cells responsive to the test line might not have been adapted. Movement insured that all cells responsive to the test line in the visual field would be stimulated.

Experiment 2 was designed to check whether or not movement of the adapting field was in fact necessary to elevate the threshold of the matching test line. By positioning the test line precisely between two adjacent lines of the stationary adapting field, we insured that retinal adaptation could not account for the elevated threshold found in Experiment 2. An explanation in line with Hubel and Wiesel's conception of orientation detectors in infrahuman species is as follows: The $S$ was not required to fixate his gaze. Voluntary eye movements were certainly made and, in addition, spontaneous nystagmus must have insured that the image of the stationary adapting lines would play back and forth across the retinal mosaic. If this alternative proves to be correct, then the results obtained in Experiment 2 do, in fact, follow from the original supposition, derived from the Hubel-Wiesel 
conception, that movement is necessary to obtain the differential test thresholds seen when test and adapting lines match.

Psychophysical studies of orientation-specific mechanisms have also been reported by Andrews (1965). Gilinsky et al (1967) found that oblique test stimuli had a lower identification threshold when test and inspection gratings were matched in orientation. Our data contradict these findings.

Psychophysical studies of motion-specific mechanisms have been reported by Sekuler and Ganz (1963) and Sekuler and Pantle (1967). Their data are analogous in many respects to those reported here.

In infrahuman species, orientation-specific mechanisms have been identified at the level of striate cortex (Hubel \& Wiesel, $1962,1965)$. It remains to be shown that orientation-specific adaptation or satiation demonstrated here for the human visual system is central, not retinal. We will address ourselves to this problem in a succeeding paper.

\section{REFERENCES}

ANDREWS, D. P. Perception of contours in the central fovea. Nature, $1965,205,1218-1220$.

GILINSKY, A. S., BOY KO, E. P., \& BARAS, H. L. Resolution of lines with specific orientations after patterned-light adaptation (Abstract). Journal of the Optical Society of America, 1967, 57, 29.
HUBEL, D. H., \& WIESEL, T. N. Receptive fields, binocular interaction and functional architecture in the cat's visual cortex. Journal of Physiology, 1962, 160, 106-154.

HUBEL, D. H., \& WIESEL, T. N. Receptive fields and functional architecture in two non-striate visual areas (18 and 19) of the cat. Journal of Neurophysiology, 1965, 28, 229-289.

SEKULER, R. W., \& GANZ, L. Aftereffect of seen motion with a stabilized retinal image. Science, 1963, 139, 419-420.

SEKULER, R. W., \& PANTLE, A. A model for after-effects of seen movement. Vision Research, 1967, 7, 427-439.

WINER, B. J. Statistical principles in experimental design. New York: McGraw-Hill, 1962. Pp. 105-116.

\section{NOTES}

1. Supported in part by United States Public Health Service Grant No. MH-03830, by 5-505-FR-07064-03 (General Research Support) and by State of Illinois Mental Health Fund Grant No. 1711.

2. Address: Institute for Psychosomatic and Psychiatric Research and Training, Michael Reese Hospital and Medical Center, 29th Street and Ellis Avenue, Chicago, Illinois 60606.

3. Lines of 45 -deg oblique orientation were omitted as an adapting stimulus. The 27 combinations of adapting and test stimuli in Experiment 1 resulted in a procedure that was tiring to the $S$. In addition, to judge from the results of Experiment 1, no single adapting field had any unique effect. Rather, its effect was derived solely as a function of the similarity or difference of its orientation from that of the test stimulus.

(Accepted for publication March 5, 1969.) 